VOL. $61(2000) \quad[189-200]$

\title{
SHARP CONSTANTS IN HIGHER-ORDER HEAT KERNEL BOUNDS
}

\section{NiCK DUNGEY}

\begin{abstract}
We consider a space $X$ of polynomial type and a self-adjoint operator on $L^{2}(X)$ which is assumed to have a heat kernel satisfying second-order Gaussian bounds. We prove that any power of the operator has a heat kernel satisfying Gaussian bounds with a precise constant in the Gaussian. This constant was previously identified by Barbatis and Davies in the case of powers of the Laplace operator on $\mathbf{R}^{N}$. In this case we prove slightly sharper bounds and show that the above-mentioned constant is optimal.
\end{abstract}

\section{INTRODUCTION}

In [1] Barbatis and Davies considered the problem of obtaining sharp constants in Gaussian heat kernel bounds for a class of higher order elliptic operators acting on $L^{2}\left(\mathbf{R}^{N}\right)$. In particular, they obtained the following result. Let $K_{t}^{(m)}$ denote the heat kernel for the operator $\Delta^{m / 2}$, where $\Delta=-\sum_{j=1}^{N} \partial_{j}^{2}$ is the ordinary Laplacian on $\mathbf{R}^{N}$ and $m$ is a positive even integer with $m>N$. Then for each $r>1$ there exists $c_{r}>0$, depending only on $m, N$, and $r$, such that

$$
\left|K_{t}^{(m)}(x ; y)\right| \leqslant c_{\tau} t^{-N / m} e^{-\left(b_{m} / r\right)\left(d(x ; y)^{m} / t\right)^{1 /(m-1)}} \quad x, y \in \mathbf{R}^{N}, t>0
$$

where the constant $b_{m}$ is given by

$$
b_{m}=(m-1) m^{-m /(m-1)} \sin (\pi /(2 m-2))
$$

and $d(x ; y)=\left(\sum_{j=1}^{N}\left(x_{j}-y_{j}\right)^{2}\right)^{1 / 2}$ is the Euclidean distance.

In this paper, we improve this result in two directions. In Section 2, we prove Theorem 1, which may roughly be stated as follows. Let $H$ be a nonnegative selfadjoint operator on $L^{2}(X ; \mu)$ for a measure space $(X, \mu)$ with a metric $d$ which satisfies a uniform condition of polynomial growth. If the heat kernel for $H$ satisfies second-order

\section{Received 12th April, 1999}

I would like to thank Derek Robinson and Tom ter Elst for their encouragement during this work and for critically commenting on the manuscript. The proof of Theorem 1 is based on their work, notes of which they generously shared with me. Alan McIntosh made some suggestions to improve the clarity of the exposition.

Copyright Clearance Centre, Inc. Serial-fee code: 0004-9727/00 \$A2.00+0.00. 
Gaussian bounds with a factor which may be chosen arbitrarily close to $b_{2}=1 / 4$ in the exponential, then the heat kernel for $H^{m / 2}$ satisfies $m$-th order Gaussian bounds with a factor arbitrarily close to $b_{m}$ in the exponential. Thus the constant $b_{m}$ is typical for powers of a general class of self-adjoint operators. This is not clear from the analysis of [1], which uses the Fourier theory of $L^{2}\left(\mathbf{R}^{N}\right)$.

The second-order Gaussian bounds with a factor arbitrarily close to 1/4 are characteristic for a variety of second-order elliptic, or subelliptic, differential operators over manifolds. For example, second-order uniformly elliptic operators in divergence form with real measurable symmetric coefficients on $\mathbf{R}^{N}$, and left-invariant sublaplacians on Lie groups of polynomial growth, satisfy the assumptions of Theorem $1[3,6,8]$.

Robinson and ter Elst showed in unpublished work that Gaussian bounds for powers of an operator may be deduced from second-order Gaussian bounds for the operator itself. A similar result, under different hypotheses, was proved by Saloff-Coste [7]. Robinson and ter Elst's proof used a Cauchy integral representation for the semigroup $S_{t}^{(m)}=$ $e^{-t H^{m / 2}}$ together with a partial fraction decomposition of the resolvent of $H^{m / 2}$ in terms of the resolvent of $H$ (a similar decomposition was previously used in [5]). Our proof of Theorem 1 follows their method, but in order to obtain the sharp constant $b_{m}$ we need more precise bounds on the kernel of the resolvent (see Lemma 5 below) and more careful choices of certain parameters.

In Section 3 we return to the special case of the operator $\Delta^{m / 2}$ on $\mathbf{R}^{N}$. Using Fourier theory we prove that (1) holds with $r=1$, and for all $m$ and $N$ without the restriction $m>N$ of [1]. Finally we confirm the conjecture of [1] that the constant $b_{m}$ is optimal, by showing that the bounds (1) cannot hold when $0<r<1$.

\section{POWERS OF SELF-ADJOINT OPERATORS}

Let $(X, d)$ be a metric space and $\mu$ a positive measure on $X$. We assume that the ball $B(x ; r)=\{y \in X: d(x ; y)<r\}$ is $\mu$-measurable for each $x \in X$ and $r>0$, and set $V(x ; r)=\mu(B(x ; r))$. We further assume that the space has uniform polynomial growth, in the sense that there are integers $D^{\prime} \geqslant 1$ and $D \geqslant 0$ such that

$$
\begin{aligned}
& C^{-1} r^{D^{\prime}} \leqslant V(x ; r) \leqslant C r^{D^{\prime}}, \quad 0<r \leqslant 1, \\
& C^{-1} r^{D} \leqslant V(x ; r) \leqslant C r^{D}, \quad r \geqslant 1,
\end{aligned}
$$

for some $C>0$ and all $x$. (The integers $D^{\prime}$ and $D$ are often called the dimensions at zero and infinity respectively.) Then $\mu$ is $\sigma$-finite, because $X=\bigcup_{n=1}^{\infty} B\left(x_{0} ; n\right)$ is a countable union of balls. The volume growth of balls is measured by the function $V$ defined by $V(r)=r^{D^{\prime}}$ or $V(r)=r^{D}$ according as $0<r<1$ or $r \geqslant 1$.

Let $H$ be a nonnegative self-adjoint operator on $L^{2}=L^{2}(X ; \mu)$. Then $H$ generates a holomorphic semigroup $S_{z}=e^{-z H}$ on $L^{2}$, defined for all $z \in \mathrm{C}$ with $\operatorname{Re} z>0$. We 
assume that $S_{t}$ has a continuous kernel $K_{t}: X \times X \rightarrow \mathrm{C}$ for each $t>0$ which satisfies Gaussian bounds with a factor arbitrarily close to $1 / 4$ in the exponential. That is,

$$
\left(S_{t} f\right)(x)=\int_{X} d \mu(y) K_{t}(x ; y) f(y), \quad f \in L^{2},
$$

and for each $r>1$ there exists $c_{r}>0$ such that

$$
\left|K_{t}(x ; y)\right| \leqslant c_{r} V(t)^{-1 / 2} e^{-d^{2} /(4 r t)}
$$

for all $t>0$ and $x, y \in X$. Here, as elsewhere, we abbreviate $d(x ; y)$ as $d$. Let $m$ be a positive even integer. Then the operator $H^{m / 2}$ is nonnegative self-adjoint on $L^{2}$ and generates a semigroup $S_{t}^{(m)}=e^{-t H^{m / 2}}$ on $L^{2}$.

THEOREM 1. Suppose that $(X, d, \mu)$, and $H$ acting on $L^{2}(X ; \mu)$, satisfy the above assumptions, and let $m \geqslant 4$ be an even integer. Then the semigroup $S_{t}^{(m)}=e^{-t H^{m / 2}}$ has an integral kernel $K_{t}^{(m)}$. Moreover for each $r>1$ there exists $c_{r}^{\prime}>0$, depending on $(X, d, \mu), H, m$, and $r$, such that

$$
\left|K_{t}^{(m)}(x ; y)\right| \leqslant c_{r}^{\prime} V(t)^{-1 / m} e^{-\left(b_{m} / r\right)}\left(d^{m} / t\right)^{1 /(m-1)}, \quad d=d(x ; y),
$$

for all $t>0$ and $x, y \in X$.

The first step in the proof of Theorem 1 is to derive uniform bounds.

Lemma 2. The semigroup $S_{t}^{(m)}$ has an integral kernel $K_{t}^{(m)}$ satisfying bounds

$$
\left|K_{t}^{(m)}(x ; y)\right| \leqslant c V(t)^{-1 / m}
$$

for all $t>0$ and $x, y \in X$.

Proof: Let $\|\cdot\|_{p \rightarrow q}$ denote the norm of a bounded linear operator from $L^{p}(X ; \mu)$ to $L^{q}(X ; \mu)$. Then

$$
\left\|S_{t}\right\|_{2 \rightarrow \infty} \leqslant \sup _{x \in X}\left(\int d \mu(y)\left|K_{t}(x ; y)\right|^{2}\right)^{1 / 2} \leqslant c V(t)^{-1 / 4}
$$

where the second inequality follows from the Gaussian bounds on $K$ by a quadrature argument (see for example [4, Proposition 2.1]). Fix $k>N / 2$, where $N=D^{\prime} \vee D$. For each $\rho>0$ one has the identity

$$
(I+\rho H)^{-k / 2}=\Gamma(k / 2)^{-1} \int_{0}^{\infty} d t e^{-t} t^{-1+(k / 2)} S_{t \rho}
$$

and using a volume inequality $V(t \rho)^{-1 / 4} \leqslant c\left(1+t^{-N / 4}\right) V(\rho)^{-1 / 4}$ one finds that

$$
\left\|(I+\rho H)^{-k / 2}\right\|_{2 \rightarrow \infty} \leqslant c^{\prime} V(\rho)^{-1 / 4} \int_{0}^{\infty} d t e^{-t} t^{1+(k / 2)}\left(1+t^{-N / 4}\right)=c^{\prime \prime} V(\rho)^{-1 / 4}
$$


for all $\rho>0$. Using this estimate and spectral theory gives

$$
\left\|S_{t}^{(m)}\right\|_{2 \rightarrow \infty} \leqslant\left\|\left(I+t^{2 / m} H\right)^{-k / 2}\right\|_{2 \rightarrow \infty}\left\|\left(I+t^{2 / m} H\right)^{k / 2} S_{t}^{(m)}\right\|_{2 \rightarrow 2} \leqslant c^{\prime} V(t)^{-1 /(2 m)} .
$$

Therefore

$$
\left\|S_{t}^{(m)}\right\|_{1 \rightarrow \infty} \leqslant\left(\left\|S_{t / 2}^{(m)}\right\|_{2 \rightarrow \infty}\right)^{2} \leqslant c V(t)^{-1 / m}
$$

and the lemma follows by the Dunford-Pettis Theorem.

To derive Gaussian bounds, following an unpublished argument of ter Elst and Robinson, we first reduce to the case where $D^{\prime}=D \geqslant 4$.

LEMMA 3. If Theorem 1 holds when $D^{\prime}=D \geqslant 4$, then it holds generally.

Proof: Suppose that the quadruple $(X, d, \mu, H)$ satisfies the assumptions of Theorem 1. If $D^{\prime}>D$ define $X_{2}=G^{D^{\prime}-D} \times \mathrm{R}^{3}$ where $G$ is the three-dimensional Heisenberg group, if $D^{\prime}<D$ define $X_{2}=\mathrm{T}^{D-D^{\prime}} \times \mathbf{R}^{3}$ and if $D^{\prime}=D$ define $X_{2}=\mathbf{R}^{3}$. Then $X_{2}$ is a Lie group and we let $\mu_{2}$ be the (bi-invariant) Haar measure on $X_{2}$. Choose left-invariant vector fields $A_{1}, \ldots, A_{k}$ which form a vector space basis for the Lie algebra of $X_{2}$, and let $d_{2}$ be the left-invariant distance and $H_{2}=-\sum_{j=1}^{k} A_{j}^{2}$ the Laplacian associated with this choice. Then $\left(X_{2}, d_{2}, \mu_{2}, H_{2}\right)$ satisfies the assumptions of Theorem 1 ; in particular, the kernel $K_{2, t}$ of $e^{-t H_{2}}$ satisfies Gaussian bounds with a factor arbitrarily close to $1 / 4[6,8]$. If $D_{2}^{\prime}$ and $D_{2}$ are the dimensions at zero and infinity of $\left(X_{2}, d_{2}, \mu_{2}\right)$ then $D_{2}^{\prime}+D^{\prime}=D_{2}+D \geqslant 4$. Moreover, since $H_{2}^{m / 2} 1=0$ it follows that $1=\left(S_{2, t}^{(m)} 1\right)\left(x_{2}\right)=\int_{X_{2}} d \mu_{2}\left(y_{2}\right) K_{2, t}^{(m)}\left(x_{2} ; y_{2}\right)$ for all $x_{2} \in X_{2}$, where $K_{2, t}^{(m)}$ is the kernel of $S_{2, t}^{(m)}=e^{-t H_{2}^{m / 2}}$.

Now define $\widetilde{X}=X \times X_{2}$ and let $\tilde{d}\left(\left(x, x_{2}\right) ;\left(y, y_{2}\right)\right)^{2}=d(x ; y)^{2}+d_{2}\left(x_{2} ; y_{2}\right)^{2}$ for $\left(x, x_{2}\right)$, $\left(y, y_{2}\right) \in \widetilde{X}$. Let $\tilde{\mu}=\mu \times \mu_{2}$ be the product measure on $\widetilde{X}$, and set $\widetilde{H}=H \otimes I+I \otimes H_{2}$, where we have identified $L^{2}(\widetilde{X})=L^{2}(X) \otimes L^{2}\left(X_{2}\right)$. Then the quadruple $(\widetilde{X}, \tilde{d}, \tilde{\mu}, \widetilde{H})$ satisfies the assumptions of Theorem 1 , and moreover the dimensions at zero and infinity of $(\widetilde{X}, \tilde{d}, \tilde{\mu})$ are equal and not less than 4 . Thus by assumption, the kernel $\widetilde{K}_{t}^{(m)}$ of $\tilde{S}_{t}^{(m)}=e^{-t \widetilde{H}^{m / 2}}$ satisfies Gaussian bounds with a factor arbitrarily close to $b_{m}$. One easily sees that

$$
\widetilde{K}_{t}^{(m)}\left(\left(x, x_{2}\right) ;\left(y, y_{2}\right)\right)=K_{t}^{(m)}(x ; y) K_{2, t}^{(m)}\left(x_{2} ; y_{2}\right)
$$

for all $x, y \in X$ and $x_{2}, y_{2} \in X_{2}$. Since $\int_{X_{2}} d \mu_{2}\left(y_{2}\right) K_{2, t}^{(m)}\left(x_{2} ; y_{2}\right)=1$ we obtain

$$
K_{t}^{(m)}(x ; y)=\int_{X_{2}} d \mu_{2}\left(y_{2}\right) \widetilde{K}_{t}^{(m)}\left(\left(x, x_{2}\right) ;\left(y, y_{2}\right)\right)
$$

But for any $r>1$ and $r^{\prime} \in(1, r)$, the kernel $\widetilde{K}^{(m)}$ satisfies bounds

$$
\begin{aligned}
\left|\widetilde{K}_{t}^{(m)}\left(\left(x, x_{2}\right) ;\left(y, y_{2}\right)\right)\right| & \leqslant c_{r^{\prime}} \tilde{V}(t)^{-1 / m} e^{-\left(b_{m} / r^{\prime}\right)\left(\widetilde{d^{m}} / t\right)^{1 /(m-1)}} \\
& \leqslant c_{r^{\prime}} V(t)^{-1 / m} e^{-\left(b_{m} / r\right)\left(d^{m} / t\right)^{1 /(m-1)}} V_{2}(t)^{-1 / m} e^{-\varepsilon\left(d_{2}^{m} / t\right)^{1 /(m-1)}}
\end{aligned}
$$


where $\varepsilon=\left(b_{m} / r^{\prime}\right)-\left(b_{m} / r\right)>0$. Integrating these bounds over $X_{2}$ with respect to $y_{2}$ yields Gaussian bounds on $K_{t}^{(m)}$ with a factor of $b_{m} / r$, as required.

In the remainder of the proof of Theorem 1 we shall assume that $D^{\prime}=D \geqslant 4$, so that $V(r)=r^{D}$ for all $r>0$.

LEMMA 4. The operator $S_{z}=e^{-z H}$ has a kernel $K_{z}$ satisfying bounds

$$
\left|K_{z}(x ; y)\right| \leqslant c_{r}(\operatorname{Re} z)^{-D / 2} e^{-\operatorname{Re}\left\{d^{2} /(4 r z)\right\}}=c_{r}|z|^{-D / 2}(\cos \theta)^{-D / 2} e^{-\cos \theta d^{2} /(4 r|z|)}
$$

for all $z \in \mathrm{C}$ with $\operatorname{Re} z>0$ and $\theta=\arg z$, all $r>1$ and all $x, y \in X$.

PROOF: The existence of the kernel $K_{z}$, and uniform bounds on $K_{z}$, follow from bounds

$$
\begin{aligned}
\left\|e^{-z H}\right\|_{1 \rightarrow \infty} & \leqslant\left\|e^{-(t / 2) H}\right\|_{2 \rightarrow \infty}\left\|e^{-i s H}\right\|_{2 \rightarrow 2}\left\|e^{-(t / 2) H}\right\|_{1 \rightarrow 2} \\
& \leqslant\left(\left\|e^{-(t / 2) H}\right\|_{2 \rightarrow \infty}\right)^{2} \leqslant c t^{-D / 2}=c(\operatorname{Re} z)^{-D / 2}
\end{aligned}
$$

where $z=t+i s$ with $t>0, s \in \mathbf{R}$. Then the lemma is obtained by a complex-analytic argument as in [3, Theorem 3.4.8].

For $\lambda \in \mathbf{C}-(-\infty, 0]$ we let $R_{\lambda}(\cdot ; \cdot)$ denote the integral kernel of $(\lambda I+H)^{-1}$.

LEMmA 5. For any $\rho \in[0, \pi)$, and any $q>1$, there is a $c=c(\rho, q)>0$ such that

$$
\left|R_{\lambda}(x ; y)\right| \leqslant c d^{-D+2} e^{-|\lambda|^{1 / 2} q^{-1} \cos (\theta / 2) d}
$$

for all $\lambda \in \mathbf{C}-\{0\}$ with $\theta=\arg \lambda \in[-\rho, \rho]$ and all $x, y \in X$.

PROOF: Write $\lambda=R e^{i \theta}$ where $R>0, \theta \in[0, \rho]$. (Because of the reflection relation $R_{\bar{\lambda}}(x, y)=\overline{R_{\lambda}(y, x)}$, it is sufficient to prove the lemma for such $\theta$.) Let $\tau \in[0, \pi / 2)$ be such that $0 \leqslant \theta-\tau<\pi / 2$, and set $\lambda^{\prime}=R e^{i r}$. Then

$$
(\lambda I+H)^{-1}=e^{-i(\theta-\tau)}\left(\lambda^{\prime} I+e^{-i(\theta-\tau)} H\right)^{-1}=e^{-i(\theta-\tau)} \int_{0}^{\infty} d t e^{-\lambda^{\prime} t} S_{t e^{-i(\theta-\tau)}} .
$$

Thus applying Lemma 4, and a change of variable $s=d^{-2} t$,

$$
\begin{aligned}
\left|R_{\lambda}(x ; y)\right| \leqslant & \int_{0}^{\infty} d t\left|e^{-\lambda^{\prime} t}\right|\left|K_{t e^{-i(\theta-\tau)}}(x ; y)\right| \\
\leqslant & \int_{0}^{\infty} d t e^{-R t \cos \tau} c_{r}(t \cos (\theta-\tau))^{-D / 2} e^{-(4 r)^{-1} \cos (\theta-\tau)\left(d^{2} / t\right)} \\
= & c_{r}(\cos (\theta-\tau))^{-D / 2} d^{-D+2} \int_{0}^{\infty} d s s^{-D / 2} e^{-R d^{2} s \cos \tau-(4 r)^{-1} \cos (\theta-\tau) s^{-1}} \\
= & c_{r}(\cos (\theta-\tau))^{-D / 2} d^{-D+2} \\
& \quad \cdot \int_{0}^{\infty} d s s^{-D / 2} e^{-R d^{2} s \cos \tau-\delta(4 r)^{-1} \cos (\theta-\tau) s^{-1}} e^{-(1-\delta)(4 r)^{-1} \cos (\theta-\tau) s^{-1}}
\end{aligned}
$$

for arbitrary $r>1$ and $\delta \in(0,1)$. But for every $s>0$, one has

$$
R d^{2} s \cos \tau+\delta(4 r)^{-1} \cos (\theta-\tau) s^{-1} \geqslant(R \delta / r)^{1 / 2}(\cos \tau \cos (\theta-\tau))^{1 / 2} d
$$


and hence

$$
\begin{aligned}
&\left|R_{\lambda}(x ; y)\right| \leqslant c_{\tau}(\cos (\theta-\tau))^{-D / 2} d^{-D+2} \exp \left(-(R \delta / r)^{1 / 2}(\cos \tau \cos (\theta-\tau))^{1 / 2} d\right) \\
& \cdot \int_{0}^{\infty} d s s^{-D / 2} e^{-(1-\delta)(4 \tau)^{-1} \cos (\theta-\tau) s^{-1}} .
\end{aligned}
$$

Now choose $\tau=\theta / 2$ to maximise the function $\tau \mapsto \cos \tau \cos (\theta-\tau)$ on $[0, \theta]$. Since $r$ and $\delta$ may be chosen arbitrarily close to 1 , the lemma follows.

Henceforth we assume $x, y$ and $t>0$ are such that $d(x ; y) \geqslant t^{1 / m}$ and prove the bounds of Theorem 1 under this assumption. This will complete the proof of Theorem 1 , since the bounds for $d(x ; y) \leqslant t^{1 / m}$ follow from Lemma 2 .

Let $\sigma \in(\pi / 2, \pi)$ and $R>0$ and define the contour $\Gamma=\Gamma(R, \sigma)$ in the complex plane by $\Gamma=L_{+} \cup A \cup L_{-}$, where $L_{ \pm}=\{\lambda \in \mathbf{C}: \arg \lambda= \pm \sigma,|\lambda| \geqslant R\}$ and $A=\{\lambda \in$ C : $|\arg \lambda| \leqslant \sigma,|\lambda|=R\}$. Here $\Gamma$ is oriented to run along $L_{-}$towards the origin, then anti-clockwise around $A$ and along $L_{+}$away from the origin. Then one has the Cauchy integral representation

$$
S_{t}^{(m)}=\frac{1}{2 \pi i} \int_{\Gamma} d \lambda e^{\lambda t}\left(\lambda I+H^{n}\right)^{-1}
$$

where $n=m / 2$ (see [2, Section 2.5], or [9, Chapter IX]). If $\lambda \in \mathbf{C}-\{0\}$ and $\alpha \in(0,1)$ define $\lambda^{\alpha}=|\lambda|^{\alpha} e^{i \alpha \arg \lambda}$ and let $-\lambda_{1}, \ldots,-\lambda_{n}$ be the $n$-th roots of $-\lambda$. More precisely, let $\lambda_{k}=-e^{-\pi i / n} \lambda^{1 / n} \omega^{k}$ for $k \in\{1, \ldots, n\}$, where $\omega=e^{2 \pi i / n}$. Then one has the partial fraction decomposition

$$
\left(\lambda I+H^{n}\right)^{-1}=\left(\lambda_{1} I+H\right)^{-1} \ldots\left(\lambda_{n} I+H\right)^{-1}=\sum_{k=1}^{n} c_{k}\left(\lambda^{1 / n}\right)^{1-n}\left(\lambda_{k} I+H\right)^{-1}
$$

where one may calculate $c_{k}=-e^{-\pi i / n} \prod_{1 \leqslant l \leqslant n, l \neq k}\left(\omega^{k}-\omega^{l}\right)^{-1}$. Combining this with the Cauchy integral representation yields

$$
\left|K_{t}^{(m)}(x ; y)\right| \leqslant(2 \pi)^{-1} \sum_{k=1}^{n}\left|c_{k}\right| \int_{\Gamma} d|\lambda|\left|e^{\lambda t}\right||\lambda|^{-1+(1 / n)}\left|R_{\lambda_{k}}(x ; y)\right| .
$$

We shall use Lemma 5 to bound the right hand side. First observe that if $\lambda \in \mathbf{C}-\{0\}$ and the $\lambda_{k}$ are as above, then $\pi-\left|\arg \lambda_{k}\right| \geqslant(\pi-|\theta|) / n$, where $\theta=\arg \lambda$. Hence $\left|\arg \lambda_{k}\right| / 2 \leqslant(\pi / 2)-(\pi-|\theta|) / m$ and

$$
\cos \left(\left(\arg \lambda_{k}\right) / 2\right) \geqslant \cos (\pi / 2-(\pi-|\theta|) / m)=\sin ((\pi-|\theta|) / m) .
$$

Also, $\left|\lambda_{k}\right|=|\lambda|^{1 / n}$. Therefore by Lemma 5 , for an arbitrary $q>1$ there is an $a>0$, depending on $q$ and $\sigma$, such that

$$
\begin{aligned}
\int_{A} d|\lambda|\left|e^{\lambda t}\right||\lambda|^{-1+(1 / n)}\left|R_{\lambda_{k}}(x ; y)\right| & \leqslant a \int_{-\sigma}^{\sigma} d \theta R e^{R t \cos \theta} R^{-1+(1 / n)} d^{-D+2} e^{-q^{-1} R^{1 / m} \sin ((\pi-|\theta|) / m) d} \\
& =2 a d^{-D+2} R^{1 / n} \int_{0}^{\sigma} d \theta e^{R t \cos \theta-q^{-1} R^{1 / m} \sin ((\pi-\theta) / m) d} .
\end{aligned}
$$


Now choose $R=(q m)^{-m /(m-1)}(d / t)^{m /(m-1)}$ and use the assumptions $D \geqslant 4$ and $d \geqslant t^{1 / m}$ to obtain

(5) $\int_{A} d|\lambda|\left|e^{\lambda t}\right||\lambda|^{-1+(1 / n)}\left|R_{\lambda_{k}}(x ; y)\right| \leqslant a_{1} t^{-D / m} \int_{0}^{\sigma} d \theta e^{-(q m)^{-m /(m-1)} G(\theta)\left(d^{m} / t\right)^{1 /(m-1)}}$,

where $a_{1}$ depends on $q$ and $\sigma$, and $G(\theta)=m \sin ((\pi-\theta) / m)-\cos \theta$ for $0 \leqslant \theta \leqslant \pi$. Let $\delta \in(0,1)$ be arbitrary. To estimate the integral over $L_{ \pm}$we use Lemma 5 , (4) and our choice of $R$ :

$$
\begin{aligned}
\int_{L_{ \pm}} d|\lambda|\left|e^{\lambda t}\right||\lambda|^{-1+(1 / n)}\left|R_{\lambda_{k}}(x, y)\right| & \leqslant \int_{R}^{\infty} d \tau e^{\tau t \cos \sigma} \tau^{-1+(1 / n)} a d^{-D+2} e^{-q^{-1} \tau^{1 / m} \sin ((\pi-\sigma) / m) d} \\
& \leqslant a d^{-D+2} \exp \left(\delta\left\{R t \cos \sigma-q^{-1} R^{1 / m} \sin \left(\frac{\pi-\sigma}{m}\right) d\right\}\right) \\
& \cdot \int_{R}^{\infty} d \tau e^{(1-\delta) \tau t \cos \sigma} \tau^{-1+(1 / n)} \\
& \leqslant a d^{-D+2} \exp \left(\delta\left\{R t \cos \sigma-q^{-1} R^{1 / m} \sin \left(\frac{\pi-\sigma}{m}\right) d\right\}\right) \\
& \cdot t^{-1 / n} \int_{0}^{\infty} d \nu e^{(1-\delta) \nu \cos \sigma \nu^{-1+(1 / n)}} \\
& \leqslant a_{2} t^{-D / m} e^{-(q m)^{-m /(m-1)} \delta G(\sigma)\left(d^{m} / t\right)^{1 /(m-1)}}
\end{aligned}
$$

where $a_{2}=a \int_{0}^{\infty} d \nu e^{(1-\delta) \nu \cos \sigma} \nu^{-1+(1 / n)}$ depends on $q, \sigma$ and $\delta$. Next we minimise $G$.

LEMma 6. Let $\theta_{0}=(m-2) \pi /(2 m-2), \theta_{1}=(m+2) \pi /(2 m+2)$. Then $G(\theta) \geqslant$ $(m-1) \sin (\pi /(2 m-2))$ for all $\theta \in\left[0, \theta_{1}\right]$, with equality if and only if $\theta=\theta_{0}$.

Proof: Elementary calculations show that, for $0 \leqslant \theta \leqslant \pi, G^{\prime}(\theta)=0$ precisely if $\theta=\theta_{0}$ or $\theta=\theta_{1}$, and that $G^{\prime}(\theta)<0$ for $0 \leqslant \theta<\theta_{0}$ while $G^{\prime}(\theta)>0$ for $\theta_{0}<\theta<\theta_{1}$. Since $G\left(\theta_{0}\right)=(m-1) \sin (\pi /(2 m-2))$, the proof is complete.

Now in the path of integration $\Gamma=\Gamma(R, \sigma)=\Gamma\left((q m)^{-m /(m-1)}(d / t)^{m /(m-1)}, \sigma\right)$ we fix a choice $\sigma \in\left(\pi / 2, \theta_{1}\right]$. By combining (3), (5) and (6), and applying Lemma 6, we obtain

$$
\begin{aligned}
\left|K_{t}^{(m)}(x ; y)\right| \leqslant(2 \pi)^{-1} \sum_{k=1}^{n}\left|c_{k}\right|\left(a_{1} \sigma t^{-D / m} e^{-q^{-m /(m-1)} b_{m}\left(d^{m} / t\right)^{1 /(m-1)}}\right. & \left.+2 a_{2} t^{-D / m} e^{-q^{-m /(m-1)} \delta b_{m}\left(d^{m} / t\right)^{1 /(m-1)}}\right) \\
\leqslant & a_{3} t^{-D / m} e^{-q^{-m /(m-1)} \delta b_{m}\left(d^{m} / t\right)^{1 /(m-1)}}
\end{aligned}
$$

where $a_{3}=(2 \pi)^{-1}\left(a_{1} \sigma+2 a_{2}\right) \sum_{k=1}^{n}\left|c_{k}\right|$ depends on $q, \sigma$ and $\delta$. Since $q>1$ and $\delta \in(0,1)$ may be chosen arbitrarily close to 1 , the proof of Theorem 1 is complete.

\section{Powers of the Laplacian on $\mathbf{R}^{N}$}

If $\alpha$ is a multi-index, and $\zeta$ a vector in $\mathbf{R}^{N}$ or $\mathbf{C}^{N}$, we use the standard notations $\partial^{\alpha}$ for $\partial_{1}^{\alpha_{1}} \ldots \partial_{N}^{\alpha_{N}},|\alpha|$ for $\alpha_{1}+\cdots+\alpha_{N}$ and $\zeta^{\alpha}$ for $\zeta_{1}^{\alpha_{1}} \ldots \zeta_{N}^{\alpha_{N}}$. Moreover $|x|$ denotes the 
Euclidean norm of $x \in \mathbf{R}^{N}$. We consider the operator $H=\Delta^{m / 2}$ acting on $L^{2}\left(\mathbf{R}^{N}\right)$, where $m$ is a fixed positive even integer with $m \geqslant 4$. The symbol of $H$ is the polynomial $P(\zeta)=\left(\sum_{j=1}^{N} \zeta_{j}^{2}\right)^{m / 2}$ defined for $\zeta \in \mathrm{C}^{N}$. Then $H$ corresponds in Fourier space to multiplication by $\xi \in \mathbf{R}^{N} \mapsto P(\xi)=|\xi|^{m}$. The kernel $K^{(m)}$ of the corresponding semigroup $S_{t}=e^{-t H}$ is given by $K_{t}^{(m)}(x ; y)=L_{t}(x-y)$, where

$$
L_{t}(x)=(2 \pi)^{-N} \int_{\mathbf{R}^{N}} d \xi e^{-t P(\xi)} e^{i x \cdot \xi} \quad, \quad x \in \mathbf{R}^{N}
$$

Our aim in this section is to prove

THEOREM 7. (I) The kernel satisfies bounds

$$
\left|L_{t}(x)\right| \leqslant c t^{-N / m} e^{-b_{m}\left(|x|^{m} / t\right)^{1 /(m-1)}}
$$

for all $x \in \mathbf{R}^{N}$ and $t>0$, where $b_{m}$ is given by (2) and $c>0$ is a constant depending only on $m$ and $N$.

(II) The coefficient $b_{m}$ in these bounds is optimal, that is, the bounds are not valid if $b_{m}$ is replaced by any $b$ with $b>b_{m}$.

We shall prove part (I) first. It is convenient to introduce the function $\sigma:(0, \infty) \rightarrow$ $(0, \infty)$ defined by $\sigma(k)=m^{-1}(m-1)(k m)^{-1 /(m-1)}$. Then note that inf $\left\{k \lambda^{m} t-\lambda \rho\right.$ : $\lambda>0\}=-\sigma(k)\left(\rho^{m} / t\right)^{1 /(m-1)}$ for each $t>0, \rho \geqslant 0$ and $k>0$. Also observe that if we define

$$
k_{m}=(\sin (\pi /(2 m-2)))^{-m+1}
$$

then $\sigma\left(k_{m}\right)=b_{m}$.

In the following preliminary lemma we write $\|(s, t)\|$ for $\left(s^{2}+t^{2}\right)^{1 / 2}$.

LEMMA 8. The polynomial $Q(s, t)=\operatorname{Re}\left((s+i)^{2}+t^{2}\right)^{m / 2}, s, t \in \mathbf{R}$, has absolute minimum $-k_{m}$ achieved at precisely two points $(s, t)=\left( \pm s_{m}, 0\right)$, where $s_{m}>0$ depends only on $m$. There exist $c_{1}, c_{2}>0$ such that

$$
\begin{aligned}
& Q(s, t)=-k_{m}+c_{1}\left(s-s_{m}\right)^{2}+c_{2} t^{2}+O\left(\left\|\left(s-s_{m}, t\right)\right\|^{3}\right) \quad \text { as }(s, t) \rightarrow\left(s_{m}, 0\right), \\
& Q(s, t)=-k_{m}+c_{1}\left(s+s_{m}\right)^{2}+c_{2} t^{2}+O\left(\left\|\left(s+s_{m}, t\right)\right\|^{3}\right) \quad \text { as }(s, t) \rightarrow\left(-s_{m}, 0\right) .
\end{aligned}
$$

Moreover, for any $\delta>0$ there exists a $K_{\delta}>0$ such that

$$
Q(s, t) \geqslant-k_{m}+K_{\delta}\left(s^{2}+t^{2}\right)^{m / 2}
$$

for all $(s, t)$ such that $\left\|\left(s-s_{m}, t\right)\right\| \geqslant \delta$ and $\left\|\left(s+s_{m}, t\right)\right\| \geqslant \delta$.

Proof: To minimise $s \mapsto Q(s, 0)=\operatorname{Re}(s+i)^{m}$ one sets $s+i=\mu e^{i \theta}, \mu>0$, $0<\theta<\pi$, as in [1]. Then $\mu^{2}=\sin ^{-2} \theta$ and $Q(s, 0)=S(\theta):=\sin ^{-m} \theta \cos (m \theta)$. 
By elementary calculus one finds that $S$ achieves an absolute minimum $-k_{m}$, precisely at the points $\theta=\theta_{m}, \theta=\pi-\theta_{m}$, where $\theta_{m}=\pi /(2 m-2)$. Furthermore, $S^{\prime \prime}\left(\theta_{m}\right)=$ $S^{\prime \prime}\left(\pi-\theta_{m}\right)>0$. Thus $S(\theta)=-k_{m}+(1 / 2) S^{\prime \prime}\left(\theta_{m}\right)\left(\theta-\theta_{m}\right)^{2}+O\left(\left(\theta-\theta_{m}\right)^{3}\right)$ as $\theta \rightarrow \theta_{m}$, with a similar expression for $\theta$ close to $\pi-\theta_{m}$. Next consider $\tilde{Q}(\theta, t):=Q(s, t)$ : by expanding the brackets in the definition of $Q$, one finds that $\widetilde{Q}$ is the sum of $S(\theta)$ and terms in $t^{2}, t^{4}, \ldots, t^{m}$ whose coefficients depend on $\theta$. In particular, explicit calculation shows that the coefficient of $t^{2}$ is positive when evaluated at $\theta=\theta_{m}$ (or $\theta=\pi-\theta_{m}$ ). Upon changing back from $\theta$ to $s$, this leads to the expansions of $Q$ near $\left( \pm s_{m}, 0\right)$, where $s_{m}+i=\sin ^{-1}\left(\theta_{m}\right) e^{i \theta_{m}}$.

Next, by calculating $\partial Q / \partial s, \partial Q / \partial t$ one finds that the only stationary points $\left(s_{0}, t_{0}\right)$ of $Q$ with $t_{0} \neq 0$ are $\left(s_{0}, t_{0}\right)=(0, \pm 1)$. Since $Q(0, \pm 1)=0$ and $Q(s, t) \rightarrow \infty$ as $\|(s, t)\| \rightarrow \infty$ it follows that $-k_{m}$ is indeed the absolute minimum of $Q$.

Since $Q(s, t)$ is the sum of $\left(s^{2}+t^{2}\right)^{m / 2}$ and terms which have lower degree in $s$ and $t$, the final statement of the lemma certainly holds when $\|(s, t)\|$ is large enough, say when $\|(s, t)\| \geqslant R$. Because $Q(s, t)+k_{m}>0$ when $(s, t) \neq\left( \pm s_{m}, 0\right)$, a simple compactness argument yields the statement for $\|(s, t)\| \leqslant R$ satisfying $\left\|\left(s \pm s_{m}, t\right)\right\| \geqslant \delta$.

For any $a \in S^{N-1}=\left\{x \in \mathbf{R}^{N}:|x|=1\right\}$ define the polynomial $P_{a}$ by $P_{a}(\xi)=$ $\operatorname{Re} P(\xi+i a)$ for $\xi \in \mathbf{R}^{N}$. In [1, Lemma 7], Barbatis and Davies identified $-k_{m}$ as the minimum value of $P_{a}$. We also require lower bounds on $P_{a}$ near the points where the minimum is achieved.

Lemma 9. The function $\xi \in \mathbf{R}^{N} \mapsto P_{a}(\xi)$ has absolute minimum $-k_{m}$, attained only at the points $\pm s_{m} a$ for $s_{m}$ as in Lemma 8. Moreover there exist $\delta>0$ and $K>0$, depending only on $m$ and $N$, such that

$$
\left.\begin{array}{l}
P_{a}(\xi) \geqslant-k_{m}+K\left|\xi-s_{m} a\right|^{2}, \quad\left|\xi-s_{m} a\right| \leqslant \delta \\
P_{a}(\xi) \geqslant-k_{m}+K\left|\xi+s_{m} a\right|^{2}, \quad\left|\xi+s_{m} a\right| \leqslant \delta
\end{array}\right\}
$$

Let $F_{a}=\left\{\xi \in \mathbf{R}^{N}:\left|\xi-s_{m} a\right| \geqslant \delta,\left|\xi+s_{m} a\right| \geqslant \delta\right\}$. Then there is $K^{\prime}>0$ depending only on $m, N$ and $\delta$ such that

$$
P_{a}(\xi) \geqslant-k_{m}+K^{\prime}|\xi|^{m}
$$

for all $a \in S^{N-1}$ and $\xi \in F_{a}$.

Proof: In the case $N=1$, one has $a= \pm 1$ and $P_{ \pm 1}(\xi)=\operatorname{Re}(\xi \pm i)^{m}$, so the lemma follows by applying Lemma 8 with $s= \pm \xi$ and $t=0$.

If $N \geqslant 2$, given $a \in S^{N-1}$ one can uniquely decompose any $\xi \in \mathbf{R}^{N}$ as $\xi=s a+\xi^{\prime}$, where $s$ is real and $\xi^{\prime}$ is a vector orthogonal to $a$. Setting $t=\left|\xi^{\prime}\right|$, simple calculations show that $P_{a}(\xi)=Q(s, t),\left|\xi-s_{m} a\right|^{2}=\left(s-s_{m}\right)^{2}+t^{2},|\xi|^{2}=s^{2}+t^{2}$, et cetera, and again the required results follow from Lemma 8. 
The key to obtaining Gaussian bounds on $L_{t}$ is to shift the contour of integration in (7) (this technique was previously used in [6, Proposition I.5.3]. By Cauchy's theorem one may replace $\xi$ by $\xi+i \lambda a$ in (7), for arbitrary $\lambda>0$ and $a \in S^{N-1}$, yielding

$$
L_{t}(x)=c \int_{\mathbf{R}^{N}} d \xi e^{-t P(\xi+i \lambda a)} e^{i x \cdot \xi} e^{-\lambda a \cdot x}=c \int_{\mathbf{R}^{N}} d \xi e^{-t \lambda^{m} P((\xi / \lambda)+i a)} e^{i x \cdot \xi} e^{-\lambda a \cdot x}
$$

where $c=(2 \pi)^{-N}$. Now we apply (9) and the following consequence of (8): there is $K^{\prime \prime}>0$ such that

$$
P_{a}(\xi) \geqslant-k_{m}+K^{\prime \prime}\left|\xi \pm s_{m} a\right|^{m}
$$

whenever $\left|\xi \pm s_{m} a\right| \leqslant \delta$. Thus

$$
\begin{aligned}
& \left|L_{t}(x)\right| \leqslant c \int_{d \xi} e^{-t \lambda^{m} P_{a}(\xi / \lambda)} e^{-\lambda a \cdot x} \\
& \leqslant c \int_{\left\{\xi:\left|(\xi / \lambda)-s_{m} a\right| \leqslant \delta\right\}} d \xi e^{-t \lambda^{m} K^{\prime \prime}\left|(\xi / \lambda)-s_{m} a\right|^{m}} e^{k_{m} \lambda^{m} t-\lambda a \cdot x} \\
& \quad+c \int_{\left\{\xi:\left|(\xi / \lambda)+s_{m} a\right| \leqslant \delta\right\}} d \xi e^{-t \lambda^{m} K^{\prime \prime}\left|(\xi / \lambda)+s_{m} a\right|^{m}} e^{k_{m} \lambda^{m} t-\lambda a \cdot x} \\
& \quad+c \int_{\left\{\xi: \xi / \lambda \in F_{a}\right\}} d \xi e^{-t \lambda^{m} K^{\prime}|\xi / \lambda|^{m}} e^{k_{m} \lambda^{m} t-\lambda a \cdot x}
\end{aligned}
$$

By changes of variable $\eta=\xi-\lambda s_{m} a, \eta=\xi+\lambda s_{m} a$ in the first two integrals we obtain

$$
\left|L_{t}(x)\right| \leqslant c e^{k_{m} \lambda^{m} t-\lambda a \cdot x}\left\{2 \int_{\mathbf{R}^{N}} d \eta e^{-K^{\prime \prime} t|\eta|^{m}}+\int_{\mathbf{R}^{N}} d \xi e^{-K^{\prime} t|\xi|^{m}}\right\}=c^{\prime} t^{-N / m} e^{k_{m} \lambda^{m} t-\lambda a \cdot x} .
$$

The proof of part (I) is completed by setting $a=x /|x|$ (or letting $a \in S^{N-1}$ be arbitrary if $x=0$ ) and minimising over $\lambda>0$.

We turn to the proof of (II). Following [1], let $\mathcal{E}$ be the set of linear functions $\phi: \mathbf{R}^{N} \rightarrow \mathbf{R}$ of the form $\phi(x)=a \cdot x$, where $a \in S^{N-1}$. For $\lambda \in \mathbf{R}$ and $\phi \in \mathcal{E}$, we define perturbed operators and semigroups by $H_{\lambda \phi}=e^{-\lambda \phi} H e^{\lambda \phi}$ and $S_{t}^{\lambda \phi}=e^{-\lambda \phi} S_{t} e^{\lambda \phi}$. The crucial observation of [1] is that the operators $H_{\lambda \phi}$ are constant-coefficient differential operators and so can be analyzed using the Fourier transform.

LEMma 10. For $\phi \in \mathcal{E}$ with $\phi(x)=a \cdot x$, and all $\lambda \in \mathbf{R}$ and $t>0$,

$$
\left\|S_{t}^{\lambda \phi}\right\|_{2 \rightarrow 2}=e^{k_{m} \lambda^{m} t}
$$

PROOF: In this proof we write $P(\zeta)=\sum_{|\alpha|=m} c_{\alpha}(i \zeta)^{\alpha}$ and $H=\sum_{|\alpha|=m} c_{\alpha} \partial^{\alpha}$ for certain real constants $c_{\alpha}$. For a multi-index $\alpha$, and $f$ in Schwartz space, a straightforward calculation shows that

$$
e^{-\lambda \phi} \partial^{\alpha} e^{\lambda \phi} f=\sum_{\beta+\gamma=\alpha} c_{\beta \gamma}(\lambda a)^{\gamma}\left(\partial^{\beta} f\right), \quad c_{\beta \gamma}=\frac{(\beta+\gamma) !}{\beta ! \gamma !}
$$


where $\beta !=\beta_{1} ! \ldots \beta_{N}$ !. Hence $H_{\lambda \phi}=\sum_{|\alpha|=m} c_{\alpha} \sum_{\beta+\gamma=\alpha} c_{\beta \gamma}(\lambda a)^{\gamma} \partial^{\beta}$ so $H_{\lambda \phi}$ corresponds in Fourier space to multiplication by

$$
\sum_{|\alpha|=m} c_{\alpha} \sum_{\beta+\gamma=\alpha} c_{\beta \gamma}(\lambda a)^{\gamma}(i \xi)^{\beta}=\sum_{|\alpha|=m} c_{\alpha}(i(\xi-i \lambda a))^{\alpha}=P(\xi-i \lambda a)
$$

regarded as a function of $\xi \in \mathbf{R}^{N}$. Hence $S_{t}^{\lambda \phi}$ corresponds to multiplication by $\xi \mapsto$ $e^{-t P(\xi-i \lambda a)}$. Thus if $\lambda \neq 0$, Lemma 9 gives

$$
\left\|S_{t}^{\lambda \phi}\right\|_{2 \rightarrow 2}=\sup _{\xi \in \mathbf{R}^{N}}\left|e^{-t \lambda^{m} P((\xi / \lambda)-\mathbf{i} a)}\right|=e^{k_{m} \lambda^{m} t},
$$

and similarly $\left\|S_{t}\right\|_{2 \rightarrow 2}=1$ if $\lambda=0$.

Now suppose that $L_{t}$ satisfies Gaussian bounds with a factor $b, b>b_{m}$, replacing $b_{m}$ in the exponential. Choose $b^{\prime}$ with $b_{m}<b^{\prime}<b$ and set $\varepsilon=b-b^{\prime}$. Define $k^{\prime}>0$ by $\sigma\left(k^{\prime}\right)=b^{\prime}$ where the function $\sigma$ was introduced previously. Then $-b^{\prime}\left(\rho^{m} / t\right)^{1 /(m-1)} \leqslant$ $k^{\prime} \lambda^{m} t-|\lambda| \rho$ for all $t>0, \rho \geqslant 0$ and $\lambda \in \mathbf{R}$. Thus

$$
\begin{aligned}
\left|L_{t}(x-y)\right| & \leqslant c t^{-N / m} e^{-b^{\prime}\left(|x-y|^{m} / t\right)^{1 /(m-1)}} e^{-\varepsilon\left(|x-y|^{m} / t\right)^{1 /(m-1)}} \\
& \leqslant c t^{-N / m} e^{k^{\prime} \lambda^{m} t-|\lambda||x-y|} e^{-\varepsilon\left(|x-y|^{m} / t\right)^{1 /(m-1)}}
\end{aligned}
$$

for all $\lambda \in \mathbf{R}$ and $x, y \in \mathbf{R}^{N}$. Since $S_{t}^{\lambda \phi}$ has the kernel $K_{t}^{\lambda \phi}(x ; y)=e^{-\lambda \phi(x)} L_{t}(x-y) e^{\lambda \phi(y)}$ and $|\phi(x)-\phi(y)| \leqslant|x-y|$ we obtain

$$
\left|K_{t}^{\lambda \phi}(x ; y)\right| \leqslant c t^{-N / m} e^{k^{\prime} \lambda^{m} t} e^{-\varepsilon\left(|x-y|^{m} / t\right)^{1 /(m-1)}},
$$

and it follows that

$$
\left\|S_{t}^{\lambda \phi}\right\|_{\infty \rightarrow \infty}=\sup _{x \in \mathbf{R}^{N}} \int d y\left|K_{t}^{\lambda \phi}(x ; y)\right| \leqslant c^{\prime} e^{k^{\prime} \lambda^{m_{t}} t} .
$$

Here $c^{\prime}$ is a constant which does not depend on $t, \lambda$ or $\phi$. By duality, $\left\|S_{t}^{\lambda \phi}\right\|_{1 \rightarrow 1} \leqslant c^{\prime} e^{k^{\prime} \lambda^{m} t}$ and by interpolation one finds $\left\|S_{t}^{\lambda \phi}\right\|_{2 \rightarrow 2} \leqslant c^{\prime} e^{k^{\prime} \lambda^{m} t}$. But $\sigma\left(k^{\prime}\right)=b^{\prime}>b_{m}=\sigma\left(k_{m}\right)$ implies that $k^{\prime}<k_{m}$, so this contradicts Lemma 10 when $\lambda^{m} t$ is sufficiently large. Thus the Gaussian bounds with $b>b_{m}$ are impossible.

REMARK. Theorem 7 may be extended to a larger class of operators on $\mathbf{R}^{N}$. Indeed, consider a homogeneous $m$-th order operator $H=\sum_{|\alpha|=m} c_{\alpha} \partial^{\alpha}$ with constant complex coefficients $c_{\alpha}$, where $m \geqslant 4$ is even. Assume that $H$ is strongly elliptic in the sense that $\operatorname{Re} P(\xi) \geqslant \mu|\xi|^{m}, \xi \in \mathbf{R}^{N}$, for some $\mu>0$, where $P(\zeta)=\sum_{|\alpha|=m} c_{\alpha}(i \zeta)^{\alpha}, \zeta \in \mathbf{C}^{N}$, is the symbol of $H$. We define $k_{H, a}=-\min _{\xi \in \mathbf{R}^{N}} \operatorname{Re} P(\xi+i a)$ for each $a \in S^{N-1}$, and set $k_{H}=\max _{a \in S^{N-1}} k_{H, a}$. Then for each $\varepsilon>0$ there exists $\mu_{\varepsilon}>0$ such that

$$
\operatorname{Re} P(\xi+i a) \geqslant \mu_{\varepsilon}|\xi|^{m}-k_{H}-\varepsilon
$$


for all $\xi \in \mathbf{R}^{N}$ and $a \in S^{N-1}$. (For large $|\xi|$ this follows by using the strong ellipticity condition, while for small $|\xi|$ one uses the definition of $k_{H}$.) The kernel $L_{t}^{(H)}$ of $e^{-t H}$ has a Fourier representation analogous to (7) and by shifting the contour of integration as in the proof of Theorem 7 and applying (10), one obtains bounds

$$
\left|L_{t}^{(H)}(x)\right| \leqslant c_{r} t^{-N / m} e^{-\left(b_{H} / r\right)\left(|x|^{m} / t\right)^{1 /(m-1)}}
$$

for each $r>1$, where $b_{H}=\sigma\left(k_{H}\right)$. It is unclear whether one can choose $r=1$ in general: this would require a more careful analysis of the polynomials $\operatorname{Re} P(\xi+i a)$ near their minima.

The constant $b_{H}$ is optimal in the sense that the bounds (11) cannot hold if $0<r<1$. The proof of this is similar to the proof of Theorem 7(II), but in place of Lemma 10 one finds that $\left\|S_{t}^{\lambda \phi}\right\|_{2 \rightarrow 2}=e^{k_{H, a} \lambda^{m} t}$ for $\phi \in \mathcal{E}$ with $\phi(x)=a \cdot x$.

\section{REFERENCES}

[1] G. Barbatis and E.B. Davies, 'Sharp bounds on heat kernels of higher order uniformly elliptic operators', J. Operator Theory 36 (1996), 179-198.

[2] E.B. Davies, One-parameter semigroups, London Math. Soc. Monographs 15 (Academic Press, London, New York, 1980).

[3] E.B. Davies, Heat kernels and spectral theory, Cambridge Tracts in Mathematics 92 (Cambridge University Press, Cambridge, 1989).

[4] X.T. Duong and D.W. Robinson, 'Semigroup kernels, Poisson bounds, and holomorphic functional calculus', J. Funct. Anal. 142 (1996), 89-129.

[5] D.W. Robinson, 'Commutators and generators II', Math. Scand. 64 (1989), 87-108.

[6] D.W. Robinson, Elliptic operators and Lie groups, Oxford Mathematical Monographs (Oxford University Press, Oxford, 1991).

[7] L. Saloff-Coste, 'A note on Poincaré, Sobolev, and Harnack inequalities', Internat. Math. Res. Notices 2 (1992), 27-38.

[8] N.T. Varopoulos, L. Saloff-Coste and T. Coulhon, Analysis and geometry on groups, Cambridge Tracts in Mathematics 100 (Cambridge University Press, Cambridge, 1992).

[9] K. Yosida, Functional analysis (sixth edition), Grundlehren der mathematischen Wissenschaften 123 (Springer-Verlag, Berlin, Heidelberg, New York, 1980).

Centre for Mathematics and its Applications

School of Mathematical Sciences

A ustralian National University

Canberra, ACT 0200

Australia

e-mail: dungey@maths.anu.edu.au Australia 